$1-1-1999$

\title{
Partial photoionization cross section and resonance structure of $\mathrm{Br}$
}

Dong Lin

University of Central Florida

H. P. Saha

University of Central Florida

Find similar works at: https://stars.library.ucf.edu/facultybib1990 University of Central Florida Libraries http://library.ucf.edu

This Article is brought to you for free and open access by the Faculty Bibliography at STARS. It has been accepted for inclusion in Faculty Bibliography 1990s by an authorized administrator of STARS. For more information, please contact STARS@ucf.edu.

\section{Recommended Citation}

Lin, Dong and Saha, H. P., "Partial photoionization cross section and resonance structure of Br" (1999).

Faculty Bibliography 1990s. 2721.

https://stars.library.ucf.edu/facultybib1990/2721

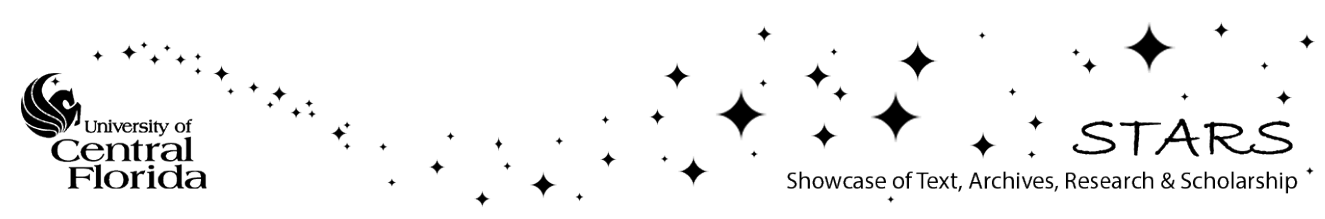




\title{
Partial photoionization cross section and resonance structure of $\mathrm{Br}$
}

\author{
Dong Lin and H. P. Saha \\ Department of Physics, University of Central Florida, Orlando, Florida 32816
}

(Received 28 September 1998)

\begin{abstract}
The partial photoionization cross sections for the outer $p$ subshell of the ground state of bromine are calculated in all available open channels as determined by $L S$ coupling for photon energies from the ${ }^{3} P$ to ${ }^{1} S$ thresholds. The electron correlations which are important in this case have been taken into account using the multiconfiguration Hartree-Fock approximation for the bound and the continuum wave functions. The resonance structures between the ${ }^{3} P$ and ${ }^{1} D$ threshold and between the ${ }^{1} D$ and ${ }^{1} S$ threshold have been identified. In order to compare with experiment a frame transformation is applied to transform the $L S$ dipole matrix elements to $j j$ coupling. The calculated energy positions for the autoionizing resonances $4 p^{4}\left({ }^{1} D\right) m d$, $4 p^{4}\left({ }^{1} D\right) n s$ and $4 p^{4}\left({ }^{1} S\right) m d$ and $4 p^{4}\left({ }^{1} S\right) n s$ are compared with recent experimental measurements, and are found to be in excellent agreement. [S1050-2947(99)10105-7]

PACS number(s): $32.80 . \mathrm{Fb}, 32.80 . \mathrm{Dz}$
\end{abstract}

\section{INTRODUCTION}

The theory of photoionization has advanced rapidly in the last few years owing to the sophisticated experimental measurements possible with high-resolution synchrotron radiation. For closed-shell atoms in most cases, both theory and experiment produced very good results because of the simplified nature of the target. The open-shell atoms are relatively difficult because of their complicated structure and affinity for chemical reaction, and are not understood well. Most of the theoretical studies for open-shell atoms have been limited, with few exceptions, to simple approximation, at best the Hartree-Fock approximation for the total cross section. Such calculations do not take into account the important electron correlation and fine-structure effects. As a result, accurate calculation of the photoionization cross section for open shell atoms test the ability of the theoretical methods to identify and understand physically important correlation effects.

Halogens are open-shell atoms, and are one outer electron short of being the ground state of noble-gas atoms. They possess a more complex structure than the closed-shell structure of rare gases, and play significant roles in chemistry and in the upper atmosphere. Autoionization resonances between the multiplet components of the halogen atoms are observed by experiments. Correct analysis and dynamics of these resonances are very important for understanding the electronic structure of these open-shell atoms.

Very recently Benzaid et al. [1] reported a comprehensive experimental study of the partial photoionization cross sections of atomic bromine across the entire autoionization regime extending from the first ionization threshold $\mathrm{Br}^{+} 4 p^{4}\left({ }^{3} P_{2}\right)$ to the highest threshold $\mathrm{Br}^{+} 4 p^{4}\left({ }^{1} S_{o}\right)$, of the multiplet created by the ionization of a $4 p$ electron from the outer subshell. In an early experiment, Sarma and Joshi [2] performed spectroscopic studies of $\mathrm{Br}$, and reported the absorption spectrum in the vacuum ultraviolet region. Earlier yet, Ruscic, Greene and Berkowitz [3] carried out ion-yield measurements to determine the relative photoionization cross section of bromine from threshold to $900 \AA$, and identified autoionizing Rydberg series. There have been several theo- retical investigations on $\mathrm{Br}$. Among the various theoretical approaches used in the calculations are the model potential approach and Hartree-Fock and eigenchannel $R$-matrix approaches. McGuire [4] used a model potential to calculate the total photoionization cross section of bromine. Manson and Msezane [5] used the Hartree-Fock approximation, as well as the model potential approach, to calculate the partial and total photoionization cross sections. In this calculation electron correlation and resonance structure were not considered. Robicheaux and Greene [6] performed a detailed calculation for the total photoionization cross sections of bromine using the eigenchannel $R$-matrix approach. They considered both correlation and fine-structure splitting of the target, the core, and continuum states, and reported results for the total cross section across the autoionization resonances.

In this paper we present results of our theoretical investigation for the partial photoionization cross sections of the outer $4 p$ subshell of bromine employing the multiconfiguration Hartree-Fock (MCHF) method for bound [7] and continuum [8] wave functions in the energy range between the $4{ }^{4}{ }^{3} P$ and ${ }^{1} S$ thresholds. To our knowledge this is the first calculation of the partial photoionization cross sections with the inclusion of autoionization resonances over this energy region. The purpose of the present investigation is to analyze and identify the resonance structures to clarify and accurately understand the recent experimental measurements and at the same time to test the ability of the present theory to describe the important electron correlation effects in the case of photoionization of atomic bromine. We report here the identification of the $4 s^{2} 4 p^{4}\left({ }^{1} D,{ }^{1} S\right) n s, m d$ resonances arising from $4 p \rightarrow n l$ photoexcitation of the bromine atom between the ${ }^{3} P$ and ${ }^{1} S$ thresholds.

It has been demonstrated that the MCHF method is capable of producing a very good description of the initial and final states, as is required for obtaining accurate photoabsorption cross sections. This method is similar to the distorted-wave $R$-matrix approach [9] developed by Seaton and his co-workers. Our calculation is based on the multiconfiguration Hartree-Fock approach using $L S$-coupled states to separately calculate the partial photoionization cross sec- 
tion for each final $L S$-coupled ionic state. The electron correlation effects which are important in this calculation have been taken into account effectively $a b$ initio in the configuration-interaction procedure. Finally, a purely algebraic transformation [10] of the $L S$ dipole matrix elements is applied to frame transform the $L S$ results to $j j$ coupling in order to compare with experiment [1] and $R$-matrix calculations [6].

\section{THEORY}

\section{A. Photoionization cross sections}

We base our calculation on the electric dipole approximation. The spin-orbit coupling and other relativistic effects are omitted. In the dipole approximation the partial photoionization cross section as a function of photon energy $\omega$ for a transition from an initial bound state $i$ to a final state $f$ is given by

$$
\sigma(\omega)=4 \pi^{2} \alpha a_{0}^{2} \omega \sum_{f, m}\left|\left\langle\Psi_{f}|T| \Psi_{i}\right\rangle\right|^{2}
$$

where $\alpha$ is the fine-structure constant, and $a_{0}$ is the Bohr radius of the hydrogen atom. $\Psi_{i}$ and $\Psi_{f}$ are, respectively, the initial- and final-state wave functions in $L S$ coupling, and the sums run over final configurations and all magnetic quantum numbers.

The dipole transition operator $T$ is given by

$$
T=T_{L}=\sum_{j=1}^{n} z_{j}
$$

in length form, and

$$
T=T_{V}=\sum_{j=1}^{n} \frac{\nabla_{j}^{z}}{i \omega}
$$

in velocity form. The length and velocity cross section will be identical when $\Psi_{i}$ and $\Psi_{f}$ are exact solutions of the same Hamiltonian.

\section{B. MCHF wave function for a continuum state}

The MCHF wave function for a continuum state may be expressed in terms of a single continuum orbital coupled to a wave function for an $N$-electron core and the other bound $(N+1)$-electron configuration state. Let

$$
\Psi\left(\gamma_{j} L_{c} S_{c} ; N\right)=\sum_{j}^{m_{c}} \Phi\left(\gamma_{j} L_{c} S_{c} ; N\right)
$$

be a wave function describing an $N$-electron core that is an eigenstate of $L_{c}$ and $S_{c}$, in terms of $N$-electron bound configuration states $\Phi\left(\gamma_{j} L_{c} S_{c} ; N\right)$ with configuration $\gamma_{j}$ and term $L_{c} S_{c}$, mixing coefficients $a_{j}$ and a total energy $E_{c}$. Let $\phi_{k l}$ be a one-electron continuum orbital with orbital angular momentum $l$. Then a MCHF wave function for a continuum state with label $\gamma$, energy $E$, and term $L S$ may be expressed in a series of the form,

$$
\begin{aligned}
\Psi(\gamma L S ; N+1)= & \sum_{j=1}^{m_{c}} a_{j} \Phi\left(\gamma_{j} L_{c} S_{c} ; N\right) \phi_{k l} \\
& +\sum_{i=1}^{m} c_{i} \Phi\left(\gamma_{i} L S ; N+1\right),
\end{aligned}
$$

where $\Phi\left(\gamma_{j} L_{c} S_{c} ; N\right) \phi_{k l}$ represents the coupling of the $N$-electron configuration with a single electron to yield an antisymmetric configuration state for the $(N+1)$-electron system with the designated final term value and configuration $\gamma_{j} k l$. The second term is the sum of $(N+1)$-electron bound-state configuration, which are eigenstates with the same $L$ and $S$ and which are included to allow for electron correlation and the core polarization.

The MCHF method for continuum state assumes that all the radial functions describing the core are fixed along with the mixing coefficients $a_{j}$. Other bound-state radial functions may be determined variationally along with the radial function for the continuum electron. A set of radial functions $P_{i}(r), i=1, \ldots, m$, which defines the above MCHF wave function, is a solution of the coupled integro-differential equation of the form

$$
\begin{aligned}
{\left[\frac{d^{2}}{d r^{2}}+\right.} & \left.\frac{2 Z}{r}-\frac{l_{i}\left(l_{i}+1\right)}{r^{2}}\right] P_{i}(r) \\
= & \frac{2}{r}\left[Y_{i}(r) P_{i}(r)+X_{i}(r)+I_{i}(r)\right] \\
& +\sum_{i^{\prime}} \epsilon_{i i^{\prime}} P_{i^{\prime}}(r),
\end{aligned}
$$

where the off-diagonal energy parameters $\epsilon_{i i^{\prime}}$ are related to Lagrange multipliers that ensure orthogonality requirements. In Eq. (5), $(2 / r) Y_{i}(r)$ is a part of a direct potential, $(2 / r) X_{i}(r)$ is the exchange function, and $(2 / r) I_{i}(r)$ represents terms arising from interactions between the configuration states.

The bound radial functions satisfy the boundary conditions

$$
\begin{aligned}
& P_{i}(r) \rightarrow r^{l+1} \quad \text { and } \quad P_{i}(r) \rightarrow 0, \\
& r \rightarrow 0 \quad r \rightarrow \infty,
\end{aligned}
$$

in which case the diagonal energy parameter $\epsilon_{i i}$ must be an eigenvalue of the integro-differential equation for the nontrivial solutions to exist, and needs to be determined. The radial function for the continuum orbital satisfy the condition:

$$
\begin{aligned}
& P_{i}(r) \rightarrow r^{l+1}, \\
& r \rightarrow 0, \\
& P_{i}(r) \rightarrow\left(\frac{2}{\pi k}\right)^{1 / 2} \sin \left[k r-\frac{l \pi}{2}+\frac{q}{k} \ln (2 k r)+\sigma_{l}+\delta_{l}\right], \\
& r \rightarrow \infty,
\end{aligned}
$$




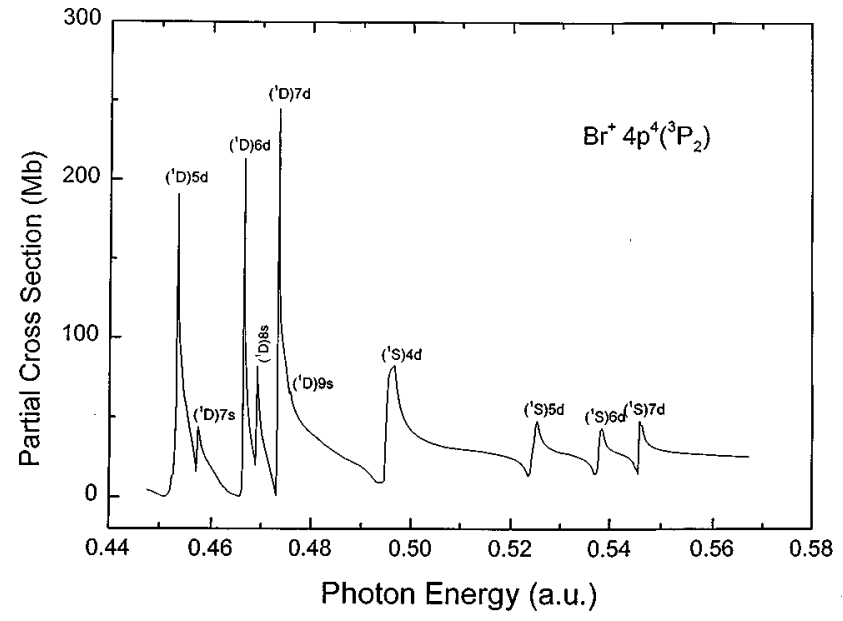

FIG. 1. Partial photoionization cross section of bromine with respect to the $\mathrm{Br}^{+} 4 p^{4}\left({ }^{3} P_{2}\right)$ core ion.

where $k^{2}$ is the kinetic energy of the photoelectron and $\epsilon_{i i}$ $=-k^{2}$. The net charge of the ion is $q=Z-N, \sigma_{l}=\arg \Gamma[l$ $+1-(i q / k)]$ is the Coulomb phase shift, and $\delta_{l}$ is the residual phase shift.

The coupled integrodifferential equations are solved numerically by an iterative method under the proper boundary conditions. The coefficients $c_{i}$ are solutions of the system of equations

$$
\sum_{i^{\prime}}^{m}\left\langle\Phi_{i}|H-E| \Phi_{i^{\prime}}\right\rangle c_{i^{\prime}}+\sum_{j=1}^{m_{c}}\left\langle\Phi_{i}|H-E| \Phi_{j}\right\rangle a_{j}=0
$$

where

$$
\Phi_{j}=\Phi\left(\gamma_{j} L_{c} S_{c} ; N\right) \phi_{k l}, \quad j=1, \ldots, m_{c}
$$

and

$$
\Phi_{i}=\Phi\left(\gamma_{i} L S ; N+1\right), \quad i=1, \ldots, m
$$

$H$ is the Hamiltonian for the $(N+1)$-electron system, and $E=E_{c}+k^{2} / 2$ in a.u.

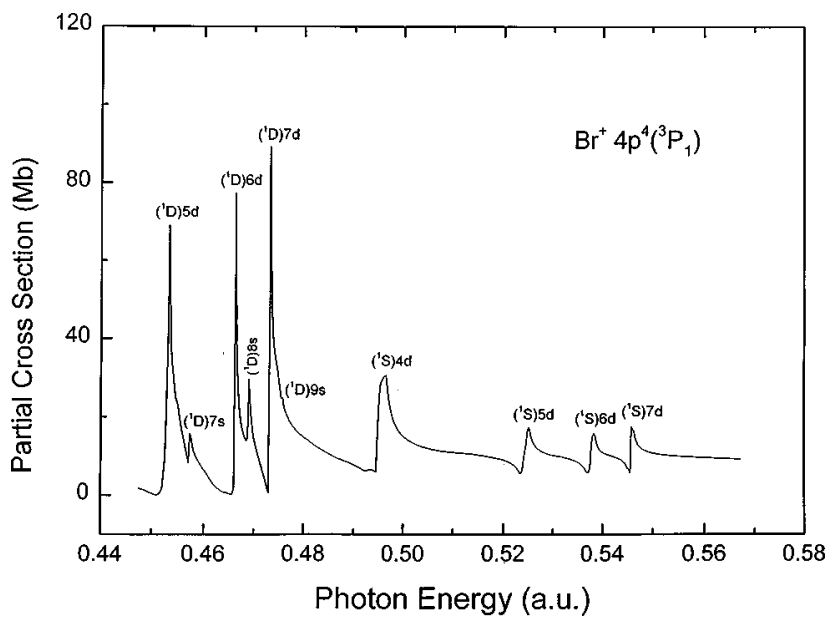

FIG. 2. Same as Fig. 1, but with respect to the $\mathrm{Br}^{+} 4 p^{4}\left({ }^{3} P_{1}\right)$ core ion.

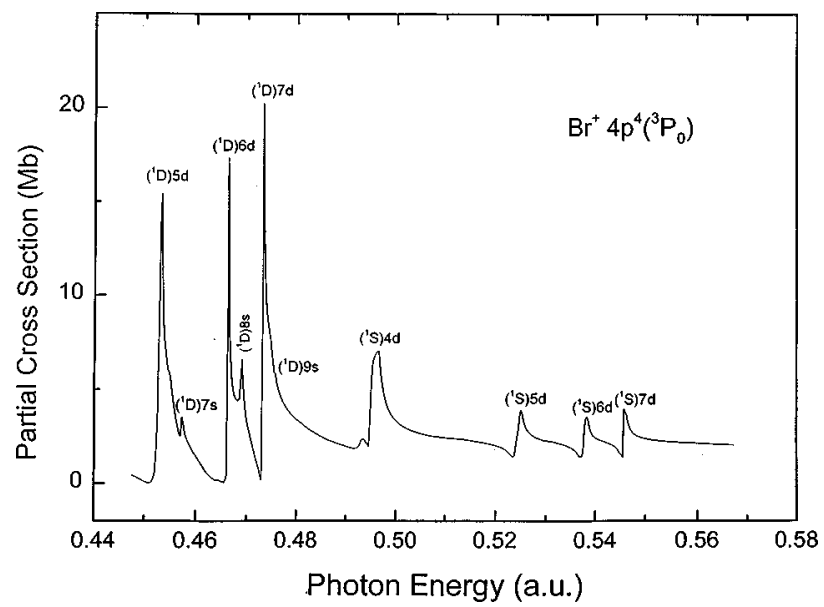

FIG. 3. Same as Fig. 1, but with respect to the $\mathrm{Br}^{+} 4 p^{4}\left({ }^{3} P_{0}\right)$ core ion.

\section{COMPUTATIONAL PROCEDURE}

The ${ }^{2} P$ ground state of bromine has a $4 s^{2} 4 p^{5}$ configuration. When a $4 p$ electron is photoionized it creates three $\left({ }^{3} P,{ }^{1} D,{ }^{1} S\right) L S$ multiplets of the bromine ion. The core ionic states ${ }^{3} P$ and ${ }^{1} D$, coupled to a $k d$ photoelectron, form $\left({ }^{2} D,{ }^{2} P\right)$ and $\left({ }^{2} S,{ }^{2} P,{ }^{2} D\right)$ final $L S$ states, respectively, and, coupled to a $k s$ photoelectron, form ${ }^{2} P$ and ${ }^{2} D$ final states. In the present investigation the photon energy considered covers the region from the ${ }^{3} P$ to ${ }^{1} S$ threshold of $\mathrm{Br}^{+}$, where the autoionization resonances appear. The following processes are considered:

$$
\begin{aligned}
\operatorname{Br}\left(4 s^{2} 4 p^{5}\right)^{2} P & \rightarrow \mathrm{Br}^{+} 4 s^{2} 4 p^{4}\left({ }^{3} P\right)+k d^{2} P,{ }^{2} D \\
& \rightarrow \mathrm{Br}^{+} 4 s^{2} 4 p^{4}\left({ }^{3} P\right)+k s^{2} P \\
& \rightarrow \mathrm{Br}^{+} 4 s^{2} 4 p^{4}\left({ }^{1} D\right)+k d^{2} S,{ }^{2} P,{ }^{2} D \\
& \rightarrow \mathrm{Br}^{+} 4 s^{2} 4 p^{4}\left({ }^{1} D\right)+k s^{2} D .
\end{aligned}
$$

Initial state: $4 s^{2} 4 p^{52} P$

First we performed a Hartree-Fock calculation for the target $4 s^{2} 4 p^{5}\left({ }^{2} P\right)$ state to determine the $1 s, 2 s, 2 p, 3 s, 3 p$,

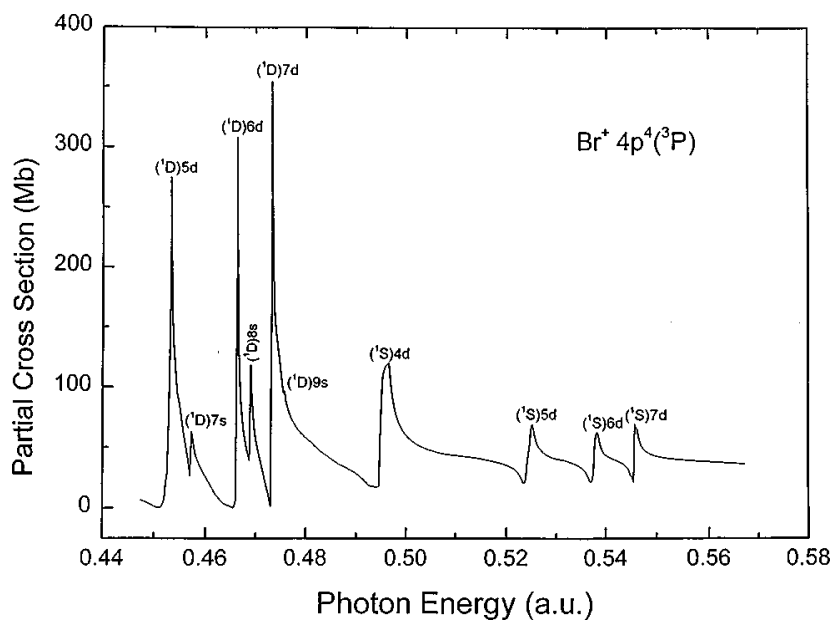

FIG. 4. Total partial photoionization cross section of bromine with respect to the ${ }^{3} P$ threshold. 
TABLE I. Comparison of threshold energies (in eV) of $\mathrm{Br}^{+} 4 p^{4}$ with experiment and observation.

\begin{tabular}{ccc}
\hline \hline State & Calculated & Experiment $^{\mathrm{a}}$ \\
\hline${ }^{3} P$ & 11.8875 & 11.9963 \\
${ }^{1} D$ & 13.1148 & 13.3127 \\
${ }^{1} S$ & 14.9135 & 15.2689 \\
\hline
\end{tabular}

${ }^{\mathrm{a}}$ References [9,10].

$3 d, 4 s$, and $4 p$ orbitals. In the MCHF approximation we expand the wave function with configurations of the same $L S$ obtained by single and double replacements of $4 s$ and $4 p$ orbitals with excited $4 d, 5 s$, and $5 p$ orbitals to take account of the electron correlation effects. We retained 40 configurations in the expansion, whose mixing coefficients are considerable. We noticed that the configurations $4 s 4 p^{5} 4 d$ and $4 s^{2} 4 p^{3} 4 d^{2}$ mix very strongly with the ground configuration.

\section{Final core states: $4 s^{2} 4 p^{43} P$ and ${ }^{1} D$}

In these calculations we performed the MCHF calculation with many configurations generated by the single and double replacements of the $4 s$ and $4 p$ orbitals with excited orbitals for each $L S$ state. It is found that in both cases the configurations $4 s 4 p^{4} 4 d, 4 s^{2} 4 p^{3} 5 p, 4 s^{2} 4 p^{3} 4 f$, and $4 s^{2} 4 p^{2} 4 d^{2}$ mix very strongly with the final core configuration. The $1 s$, $2 s, 2 p, 3 s, 3 p, 3 d, 4 s$, and $4 p$ orbitals are kept the same as the initial state.

\section{Final-state wave functions: $4 s^{2} 4 p^{4}\left({ }^{3} \mathrm{P},{ }^{1} \mathrm{D}\right) \mathrm{kl}$}

As already mentioned, there are altogether seven final $L S$ states which can be formed with the two $\left({ }^{3} P,{ }^{1} D\right)$ ionic states. We considered separately each of the final states corresponding to each of the ionic states to calculate the partial photoionization cross sections.

We first calculated the excited bound orbitals $4 d, 5 d, 6 d$, $7 d, 6 s, 7 s, 8 s, 9 s, 5 p, 6 p, 7 p$, and $8 p$ in the Hartree-Fock approximation by minimizing the energy of bromine corresponding to each of the core states and final $L S$ states. The continuum orbitals $k l$ coupled to each core state and each $L S$ final state are then determined for a range of kinetic energy of the photoelectron with a very fine energy mesh. Each final-state wave function is then expanded [Eq. (4)] in terms

TABLE II. Comparison of energies (in eV) for the $4 p^{4}\left({ }^{1} D\right) m d\left({ }^{2} D,{ }^{2} P\right)$ and $4 p^{4}\left({ }^{1} D\right) n s^{2} D$ resonances with experiment.

\begin{tabular}{cccc}
\hline \hline$m d / n s$ & $L S$ & Calculated & Experiment $( \pm 0.002)$ \\
\hline $5 d$ & ${ }^{2} D$ & 12.3615 & 12.324 \\
& ${ }^{2} P$ & 12.3669 & 12.324 \\
$7 s$ & ${ }^{2} D$ & 12.5030 & 12.429 \\
$6 d$ & ${ }^{2} D$ & 12.6935 & 12.693 \\
& ${ }^{2} P$ & 12.6880 & 12.693 \\
$8 s$ & ${ }^{2} D$ & 12.8077 & 12.752 \\
$7 d$ & ${ }^{2} D$ & 12.8295 & 12.891 \\
& ${ }^{2} P$ & 12.8159 & 12.891 \\
$9 s$ & ${ }^{2} D$ & 12.8975 & 12.927 \\
\hline \hline
\end{tabular}

TABLE III. Comparison of energies (in $\mathrm{eV}$ ) for the $4 p^{4}\left({ }^{1} S\right) m d^{2} D$ and $4 p^{4}\left({ }^{1} S\right) n s^{2} S$ resonances with experiment.

\begin{tabular}{cccc}
\hline \hline$m d / n s$ & $L S$ & Calculated & Experiment \\
\hline $4 d$ & ${ }^{2} D$ & 13.5959 & 13.485 \\
$6 s$ & ${ }^{2} S$ & 13.6948 & 13.657 \\
$5 d$ & ${ }^{2} D$ & 14.2989 & 14.283 \\
$7 s$ & ${ }^{2} S$ & 14.3723 & 14.381 \\
$6 d$ & ${ }^{2} D$ & 14.6471 & 14.653 \\
$8 s$ & ${ }^{2} S$ & 14.6852 & 14.707 \\
$7 d$ & ${ }^{2} D$ & 14.8349 & 14.850 \\
$9 s$ & ${ }^{2} S$ & 14.8539 & 14.881 \\
\hline \hline
\end{tabular}

of many excited bound and continuum configurations. The mixing coefficients $c_{i}$ are calculated by solving the algebraic equations [Eq. (9)] with the matrix elements calculated with the basis functions for the excited orbitals. In this way the wave functions for each of the final seven states are calculated for a range of kinetic energy of the photoelectron. The dipole matrix elements are then calculated in both the length and velocity approximations.

When the electron is outside the core region, the $j j$ angular coupling scheme is more appropriate than the $L S$ coupling. The dipole matrix element in $j j$ coupling can be obtained from the $L S$-coupled matrix element by a simple orthogonal transformation. The transformation matrix [10] for the final state is given by

$$
U_{L S, J J}=\left\langle\left(L_{c} l\right) L\left(S_{c} \frac{1}{2}\right) S J \mid\left(L_{c} S_{c}\right) J_{c}\left(l \frac{1}{2}\right) j J\right\rangle,
$$

which involves a Racah $9-j$ coefficient. $J_{c}$ and $J$ are the total angular momentum of the core and the final state, respectively.

\section{RESULTS}

The configuration $4 s^{2} 4 p^{4}$ of $\mathrm{Br}^{+}$has three $L S$ bound states $\left({ }^{3} P,{ }^{1} D,{ }^{1} S\right)$. In Table I we report the threshold energies (in eV) of $\mathrm{Br}^{+} 4 s^{2} 4 p^{4}$ calculated in the MCHF approximation for different $L S$ states relative to the ground state of bromine. We also tabulate the experimental energies (averaged for the ${ }^{3} P$ state) $[11,12]$ for comparison. It is found that the calculated energies are in satisfactory agreement with experiments $[11,12]$.

As already mentioned, calculations are performed separately for each of the seven allowed transitions, with the wave functions calculated separately for each of the final $L S$ states. The dipole matrix elements and hence the partial photoionization cross sections are calculated in both the length and velocity approximations. The results based on the length approximation are presented in the figures. The maximum difference between the length and velocity results is less than $10 \%$.

\section{A. $4 p \rightarrow{ }^{3} P k l$ channels}

In Figs. 1-3, the partial photoionization cross sections with respect to a specific angular momentum $J_{c}$ of the core ion $\mathrm{Br}^{+}\left({ }^{3} P\right)$ calculated in $j j$ coupling are presented for the decay channels $\mathrm{Br}^{+}\left({ }^{3} P_{2}\right) k l, \mathrm{Br}^{+}\left({ }^{3} P_{1}\right) k l$, and $\mathrm{Br}^{+}\left({ }^{3} P_{0}\right) k l$, 


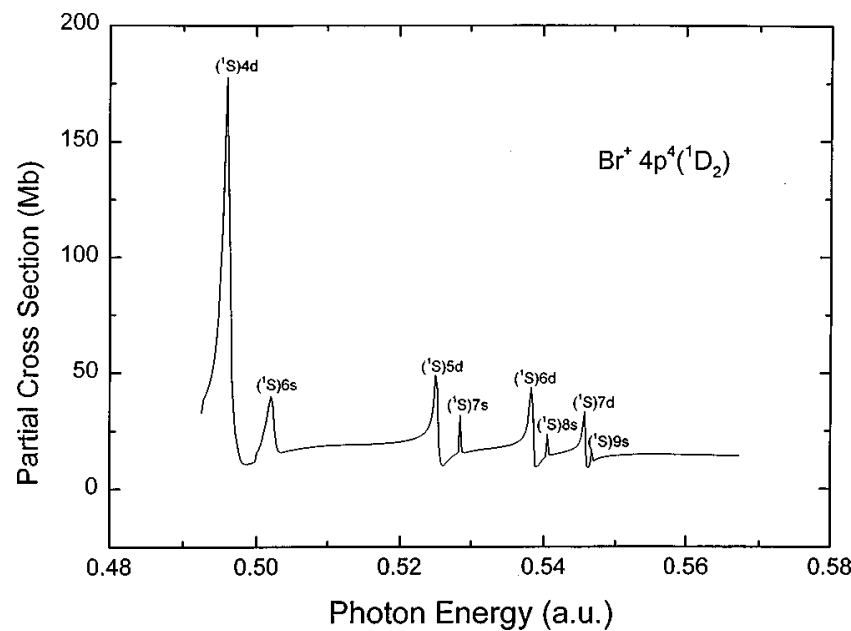

FIG. 5. Partial photoionization cross section with respect to the $\mathrm{Br}^{+}\left({ }^{1} D_{2}\right)$ core ion.

respectively. Altogether ten autoionization resonance states are found in the cross section, six resonances $4 p^{4}\left({ }^{1} D\right) m d$, $n s(m=5-7, n=6-8)$ converging to $4 p^{4}\left({ }^{1} D\right)$ and four resonances $4 p^{4}\left({ }^{1} S\right) m d(m=4-7)$ converging to $4 p^{4}\left({ }^{1} S\right)$ have been identified. Figure 4 represents the total partial cross sections summed over $J_{c}$. Energies of each of these resonances are determined and are summarized in Tables II and III. It is found that the $m d$ members are broad and $n s$ members are sharp, which is in accord with experiment. It should also be mentioned that these resonances which decay into the $k d$ continuum are much broader than those which decay into the $k s$ continuum.

\section{B. $4 p \rightarrow^{1} D k l$ channels}

In Fig. 5, the partial photoionization cross sections in $j j$ coupling with respect to the $\mathrm{Br}^{+}\left({ }^{1} D\right)$ threshold for the decay channel $\mathrm{Br}^{+}\left({ }^{1} D_{2}\right) k l$ are displayed. In this case eight resonances are identified and their energies determined. Out of these eight autoionization resonances, four resonances $4 p^{4}\left({ }^{1} S\right) m d^{2} D(m=4-7)$ decaying into the $4 p^{4}\left({ }^{1} D\right)$ $+k l^{2} D$. These resonances also appear in the $4 p \rightarrow{ }^{3} P k d$ partial cross sections, and are relatively broader than the corresponding members in the $4 p \rightarrow{ }^{3} P k d$ case. As has already been pointed out, those resonances which decay into the $k d$ continuum are broader than those which decay into the $k s$ continuum, thus following a general pattern. The other four resonances $4 p^{4}\left({ }^{1} S\right) n s(n=6-9)^{2} S$ decaying into $4 p^{4}\left({ }^{1} D\right)$ $+k d^{2} S$ are relatively small. It should be mentioned that this decay channel has a strong correlation with the $4 s 4 p^{6}\left({ }^{2} S\right)$ configuration. Figure 6 presents the total cross sections obtained with the ${ }^{3} P$ and ${ }^{1} D$ thresholds.

In Table II, we compare the energies of the autoionization resonances $4 p^{4}\left({ }^{1} D\right) m d\left({ }^{2} D,{ }^{2} P\right)$ and $4 p^{4}\left({ }^{1} D\right) n s{ }^{2} D$ found in the partial cross sections, with the recent experimental measurement [1]. The energies for the $4 p^{4}\left({ }^{1} S\right) m d^{2} D$ and $4 p^{4}\left({ }^{1} S\right) n s^{2} S$ autoionization resonances which are already identified in the partial photoionization cross sections are compared with experiment [1] in Table III. The energies of

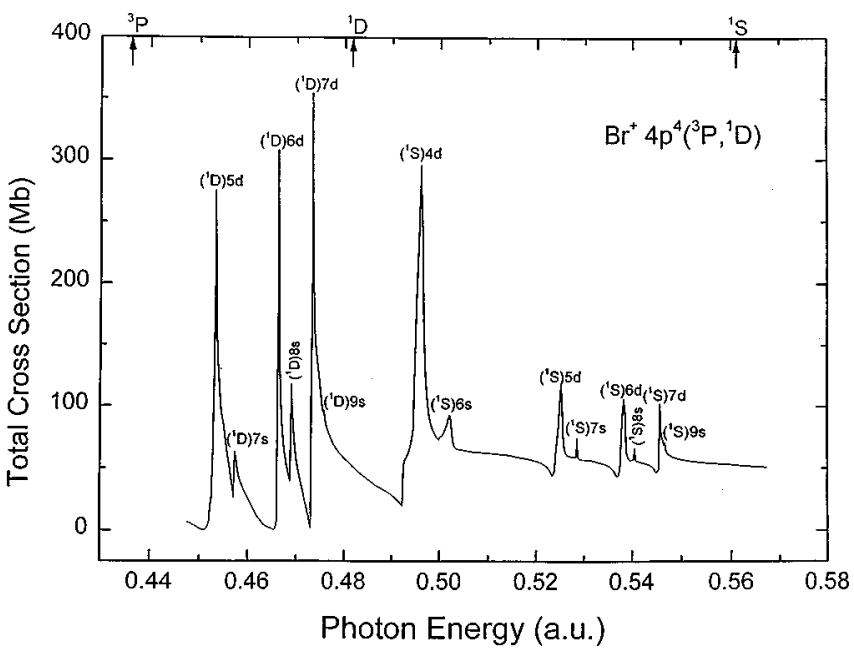

FIG. 6. Total photoionization cross section of bromine with respect to the ${ }^{3} P$ and ${ }^{1} D$ thresholds.

these autoionization resonances are found to be in good agreement with the experimental measurements [1].

Figure 7 shows the comparison of the total cross sections obtained with the MCHF method and the experiment for photon energies between the ${ }^{3} P$ and ${ }^{1} D$ thresholds. The experimental cross sections are relative and are presented in arbitrary units. The present theoretical cross sections contain six autoionization resonances converging to the ${ }^{1} D_{2}$ threshold, compared to many more structures appeared in the experimental data. Additional structures in the experimental cross sections are due to the spin-orbit interaction which is neglected in the present calculation. From the figure it is seen that the calculated resonance positions are in very good agreement with experiment. In Fig. 8, the present MCHF partial cross sections for the $\mathrm{Br}^{+} 4 p^{4}\left({ }^{3} P_{2,1,0}\right)$ are compared with experiment across the $4 p^{4}\left({ }^{1} D_{2}\right) 6 d, 8 s$ resonances. As explained earlier, additional resonances $\left({ }^{1} D_{2}\right) 6 d^{2} F$ and ${ }^{2} S$ observed in the experiment are due to spin-orbit interaction, which is not considered in the present calculation. Comparison of the sum of the cross sections for these three compo-

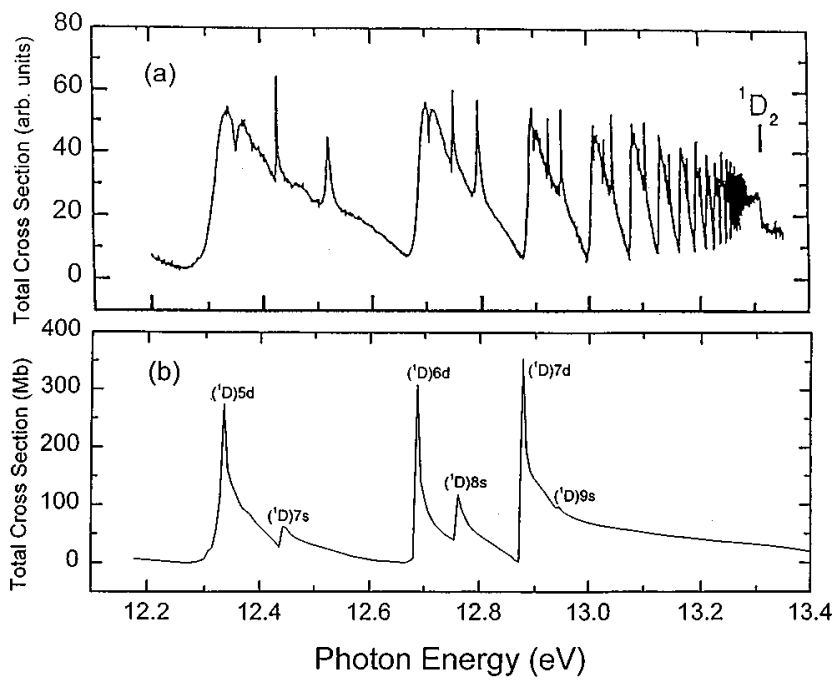

FIG. 7. Total photoionization cross section of the ${ }^{2} P_{3 / 2}$ ground state of bromine between the ${ }^{3} P$ and ${ }^{1} D$ thresholds. (a) Experimental cross sections [1]. (b) MCHF length cross section. 


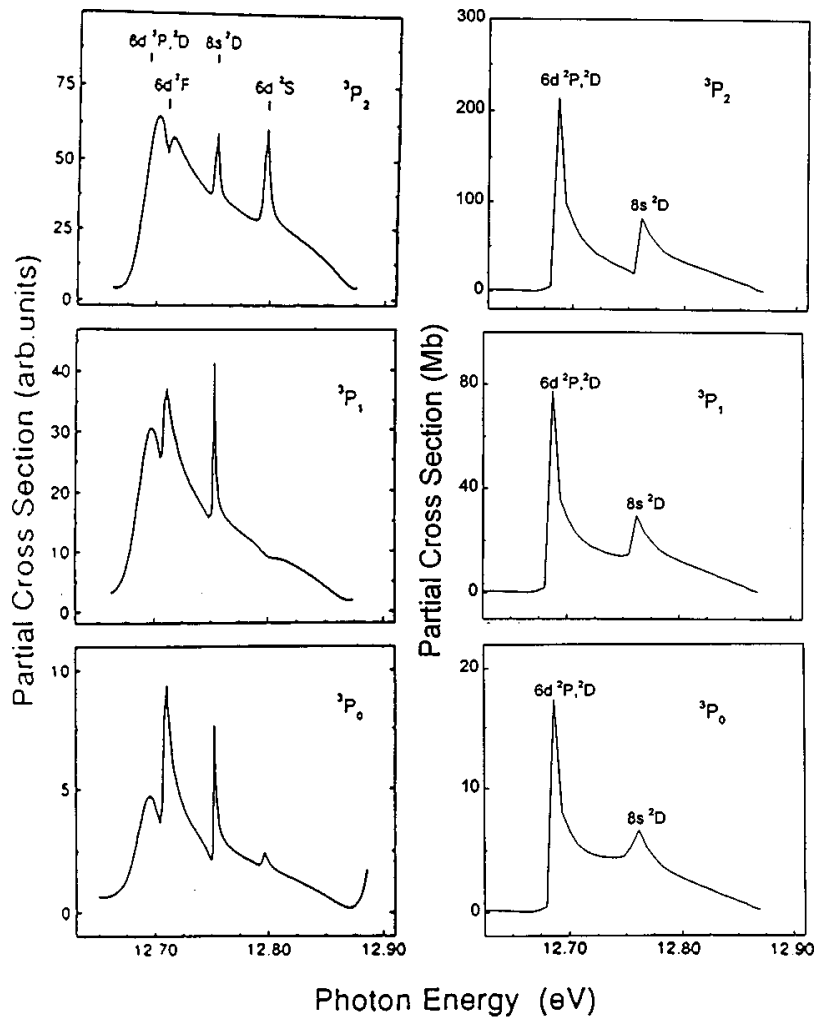

FIG. 8. Comparison of partial photoionization cross sections (right figures) for the $\mathrm{Br}^{+} 4 p^{4}\left({ }^{3} P_{2,1,0}\right)$ components with experiment (left figures) across the $4 p^{4}\left({ }^{1} D_{2}\right) 6 d, 8 s$ resonances.

nents are shown in Fig. 9. Figure 10 presents similar comparison for the total cross sections obtained in the MCHF approximation and by measurement for photon energies between the ${ }^{1} D$ and ${ }^{1} S$ thresholds. Here again, eight autoionization resonances converging to the ${ }^{1} S$ threshold are found in the present cross sections. Comparison shows that the positions of these resonances are in very good agreement with the measurement. In Fig. 11, the present MCHF partial cross

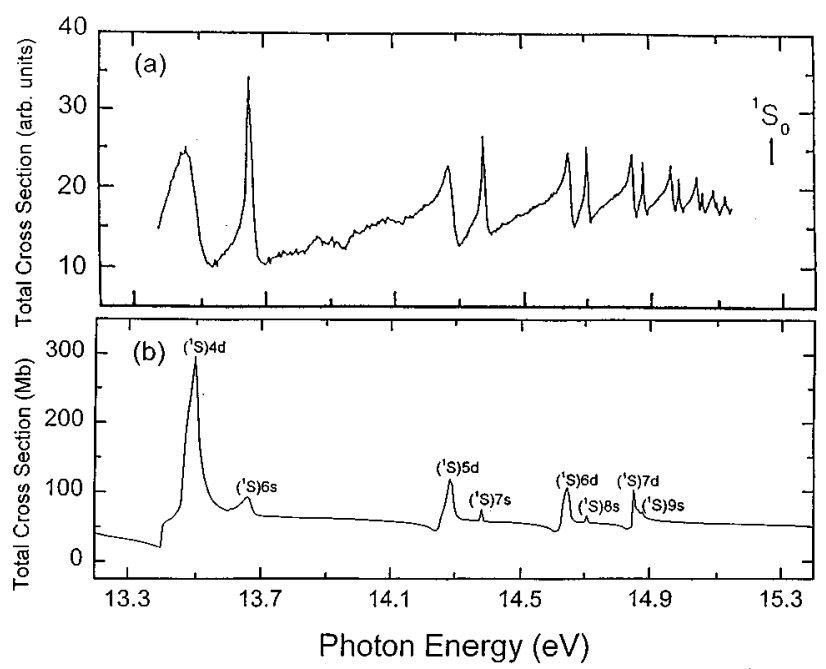

FIG. 10. Total photoionization cross section of the ${ }^{2} P_{3 / 2}$ ground state of bromine between the ${ }^{1} D$ and ${ }^{1} S$ thresholds. (a) Experimental cross sections [1]. (b) MCHF length cross sections.

sections for the $\mathrm{Br}^{+}\left({ }^{1} D_{2}\right)$ and $\mathrm{Br}^{+}\left({ }^{3} P_{2,1,0}\right)$ components are compared with experiment [1] across the $4 p^{4}\left({ }^{1} S_{o}\right) 6 d, 8 s$ resonances. Again, Fig. 12 shows the comparison of the total cross sections of these four components.

The present MCHF cross sections for photon energies between the ${ }^{3} P$ and ${ }^{1} D$ thresholds and between the ${ }^{1} D$ and ${ }^{1} S$ thresholds are compared in Figs. 13 and 14, respectively, with the corresponding theoretical results obtained by Robicheaux and Greene [6] using the eigenchannel $R$-matrix method. The $R$-matrix calculation is completely nonrelativistic; the wave function is $L S$ coupled in the numerical portion of the calculation with relativistic effects incorporated into the multichannel quantum-defect theory (MQDT) part of the calculation by applying the $L S$ to $j j$ frame transformation. The $R$-matrix cross sections presented in the figures have been convolved by the authors with experimental resolution

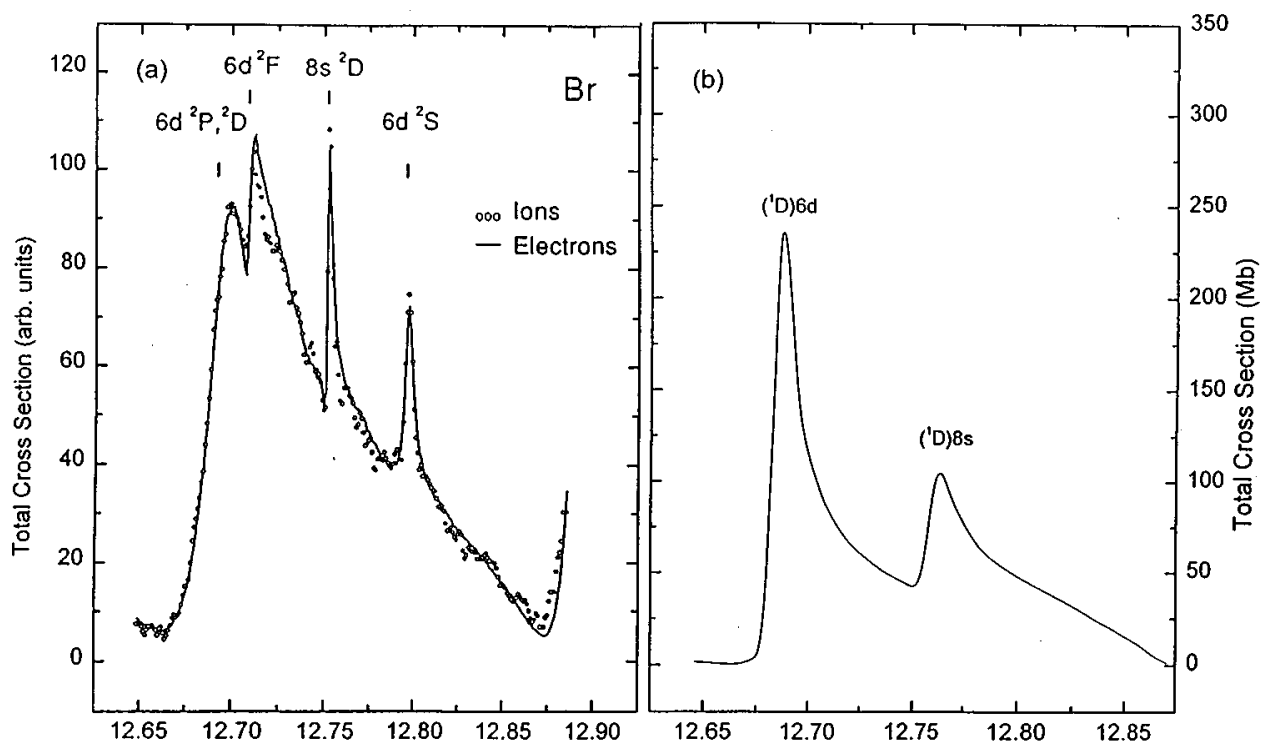

FIG. 9. Comparison of the sum of the cross sections for the three components shown in Fig. 8. (a) Experimental cross sections [1]. (b) MCHF length cross sections. 

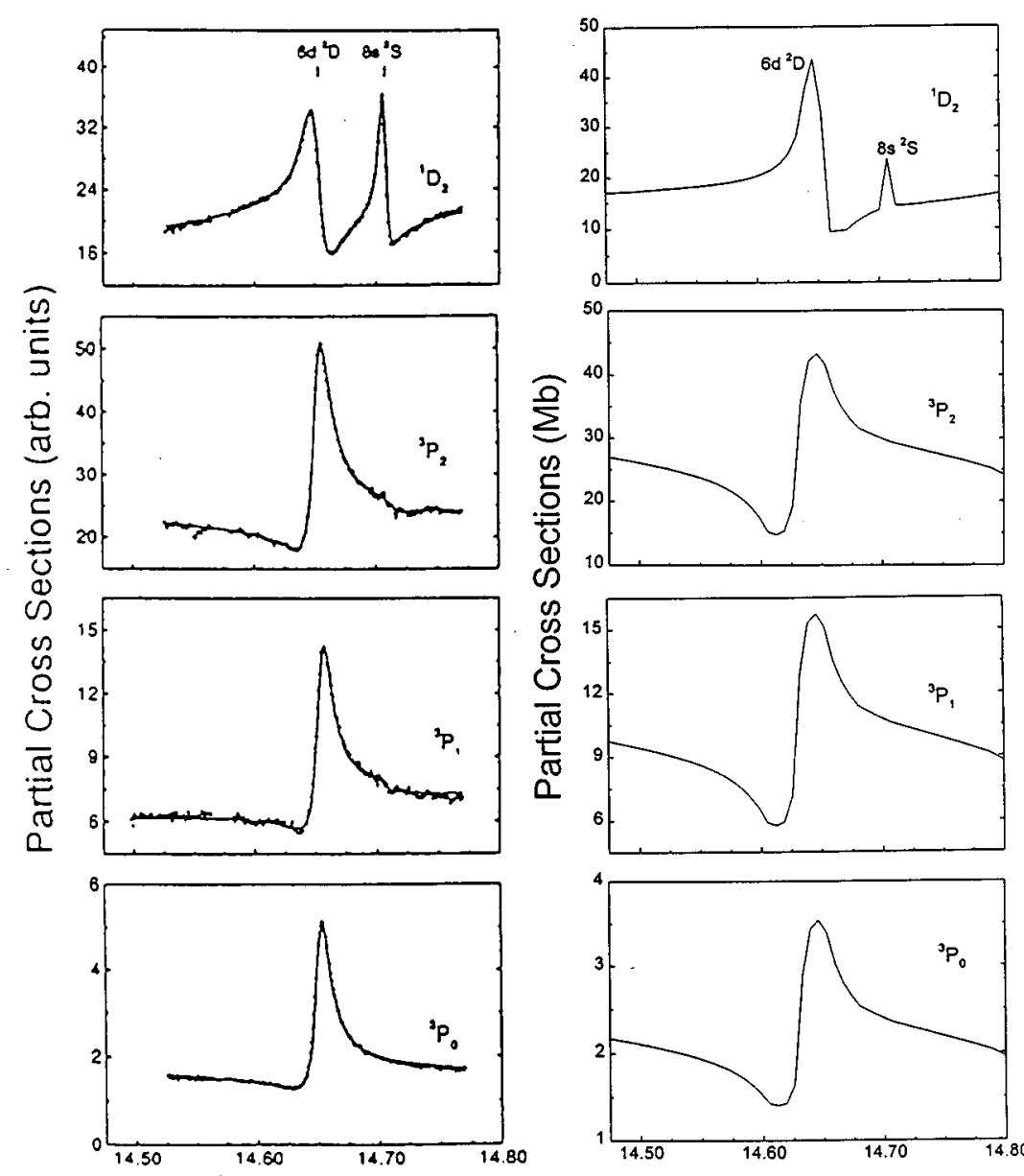

\section{Photon Energy (eV)}

of $0.28 \AA$. It appears from the figures that the present cross sections compare well with the $R$-matrix results.

\section{CONCLUSION}

We have calculated the partial photoionization cross sections into the open channels determined by $L S$ as well as $j j$ coupling for the photoionization of the $4 p$ subshell of neutral bromine for photon energies ranging from the ${ }^{3} P$ to ${ }^{1} S$ ionic threshold. Electron correlation and polarization effects, which are found to be very important in the present calculation, are considered in both the initial- and all final-state channels through the configuration interaction procedure us-
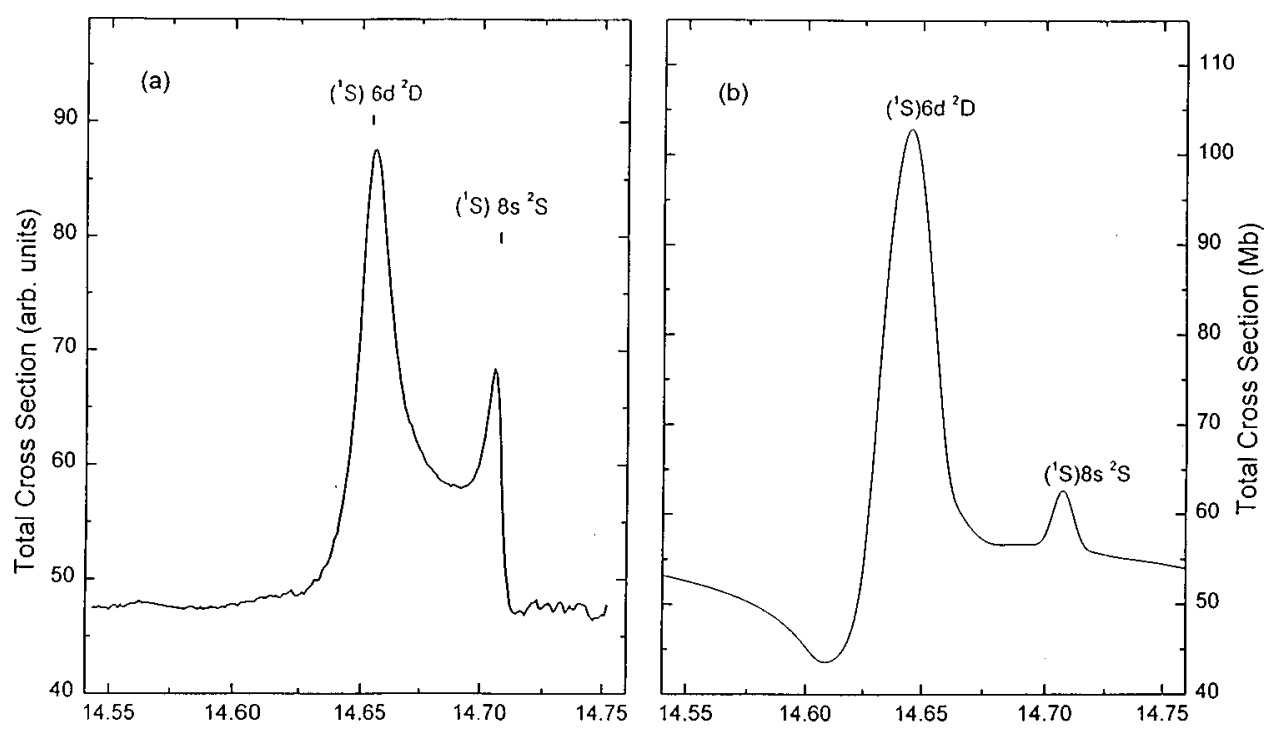

FIG. 12. Comparison of the sum of the cross sections for the four components shown in Fig. 11. (a) Experimental cross sections [1]. (b) MCHF length cross sections. 

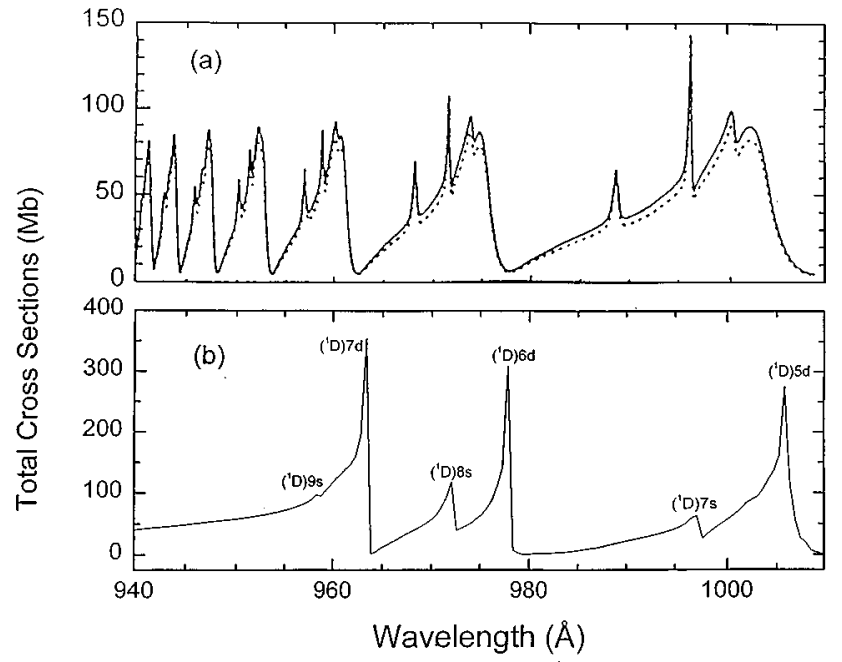

FIG. 13. Comparison of the photoionization cross section of the ${ }^{2} P_{3 / 2}$ ground state of bromine between the ${ }^{3} P$ and ${ }^{1} D$ thresholds. (a) $R$-matrix cross sections [6]. (b) MCHF length cross sections.

ing the MCHF method for bound and continuum wave functions. Our calculation shows clearly the presence of six autoionizing resonances between the ${ }^{3} P$ and ${ }^{1} D$ thresholds, and eight autoionizing resonances between the ${ }^{1} D$ and ${ }^{1} S$ thresholds. These resonances have been identified and their energy positions determined. Comparison shows that the energies of each of these resonances are in good agreement with the recent experimental measurement. In this paper we focussed on lower $n$ values for the resonance bound states,

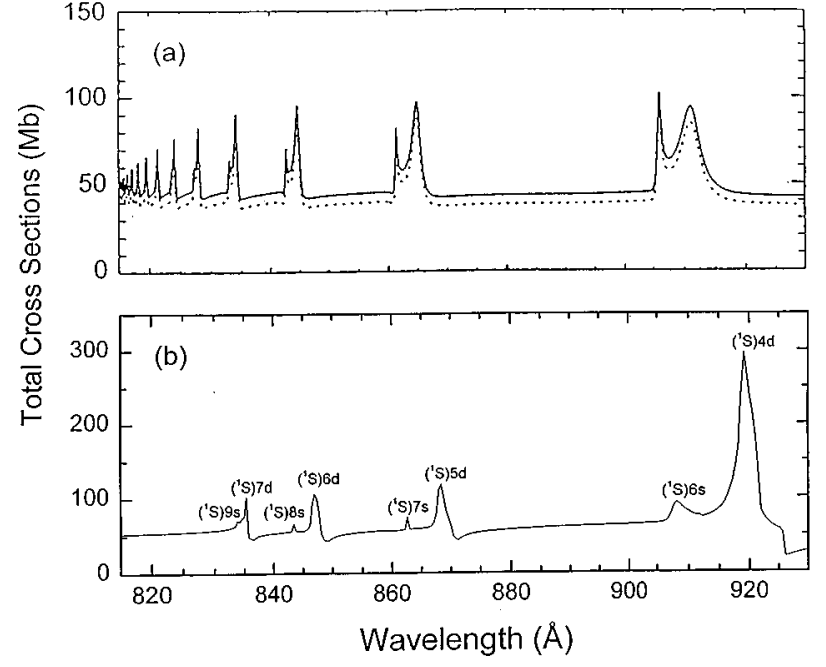

FIG. 14. Comparison of the photoionization cross section of the ${ }^{2} P_{3 / 2}$ ground state of bromine between the ${ }^{1} D$ and ${ }^{1} S$ thresholds. (a) $R$-matrix cross sections [6]. (b) MCHF length cross sections.

where the present MCHF method is found to be very useful. The extension to higher $n$ values may be made using the quantum defect theory $[13,14]$.

\section{ACKNOWLEDGMENTS}

We would like to thank Dr. F. Robicheaux for valuable discussions. This research was supported in part by the National Science Foundation.
[1] S. Benzaid, M. O. Krause, A. Menzel, and C. D. Caldwell, Phys. Rev. A 57, 4420 (1998).

[2] V. N. Sarma and Y. N. Joshi, J. Phys. B 16, 1671 (1983).

[3] B. Ruscic, J. P. Greene, and J. Berkowitz, J. Phys. B 17, 1503 (1975).

[4] E. J. McGuire, Phys. Rev. 175, 3172 (1987).

[5] S. T. Manson and E. Msezane, Phys. Rev. A 20, 1005 (1979).

[6] F. Robicheaux and C. H. Greene, Phys. Rev. A 46, 3821 (1992).
[7] C. Froese Fischer, Comput. Phys. Commun. 14, 145 (1978).

[8] H. P. Saha (unpublished).

[9] M. J. Seaton, Adv. At. Mol. Phys. 11, 83 (1975).

[10] R. D. Cowan, The Theory of Atomic Structure and Spectra (University of California Press, Berkeley, 1981), pp 248-250.

[11] J. L. Tech, J. Res. Natl. Bur. Stand., Sect. A 67A, 505 (1963).

[12] W. C. Martin and J. L. Tech, J. Opt. Soc. Am. 51, 591 (1961).

[13] M. J. Seaton, Proc. Phys. Soc. London 88, 801 (1966).

[14] M. J. Seaton, J. Phys. B 2, 5 (1969). 